\title{
Comparison of routing protocol performance on multimedia services on software defined network
}

\author{
Vivi Monita, Indrarini Dyah Irawati, Rohmat Tulloh \\ School of Applied Science, Telkom University, Indonesia
}

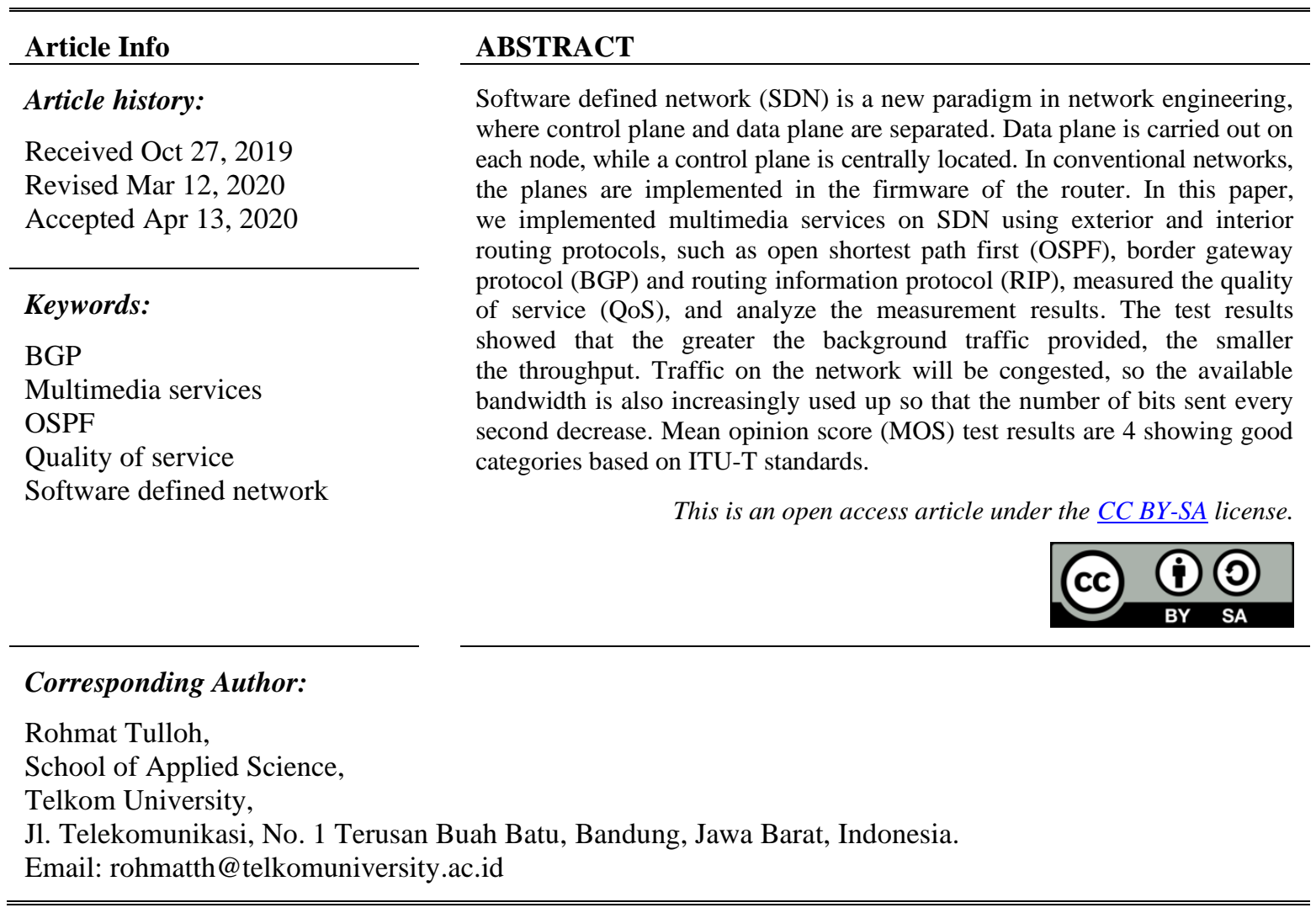

\section{INTRODUCTION}

Nowadays, multimedia applications are widely used as an effective information because it is formed by combining various kinds of datas ie. text, images, sound, video, and animation so that the information becomes clearer. Multimedia information, such as voice calls and video calls can perform remotely. This is due to the presence of digital signal processing that has modular capabilities with IP-based network that integrate data and voice communication. The software defined networks is a platform to reduce the complexity of network elements [1]. The essential ideas of SDN are the decoupling of the data plane and control plane from networks and centralized control and management of the forwarding traffic in large-scale networks [2]. The advantage of SDN is to reduce the management and maintenance costs by using the SDN controller to configure the traffic forwarding rules of SDN-enable switches dynamically in SDN [3, 4]. The OpenFlow protocol defines the communication standard in SDN environments [5-7]. Research on SDN performance with various scenarios of routing protocols or types of services has been carried out such as using the OSPF routing protocol [8-11], BGP protocol [12-14], or RIP Protocol using SDN [15-17]. The most research of SDN is usually done by simulations using simulator.

In this paper, we implemented multimedia services in the form of voice and video calls on SDN by using four switches as interconnected data planes and PC as control plane. The PC aims to control the network. We applied three routing protocols in the network, such as BGP, OSPF, and RIP. We used POX as a controller and Trixbox as a server. QoS service test on multimedia applications are performed to determine the best routing protocol. 


\section{RESEARCH METHOD}

\subsection{Software defined network}

SDN is a concept of a computer network architecture approach that controls a separate system from the hardware. In general, the current network architecture is a control system that determines the decision of the flow of data sent is still in the same device. In SDN a centralized network in a software-based controller can maintain the overall network so that it can simplify designing and operate the network because only through a logical point. SDN has three layers such as infrastructure layer (network device), control layer (network service) and the third is application layer [18]. The three layers have different functions, as in Figure 1.

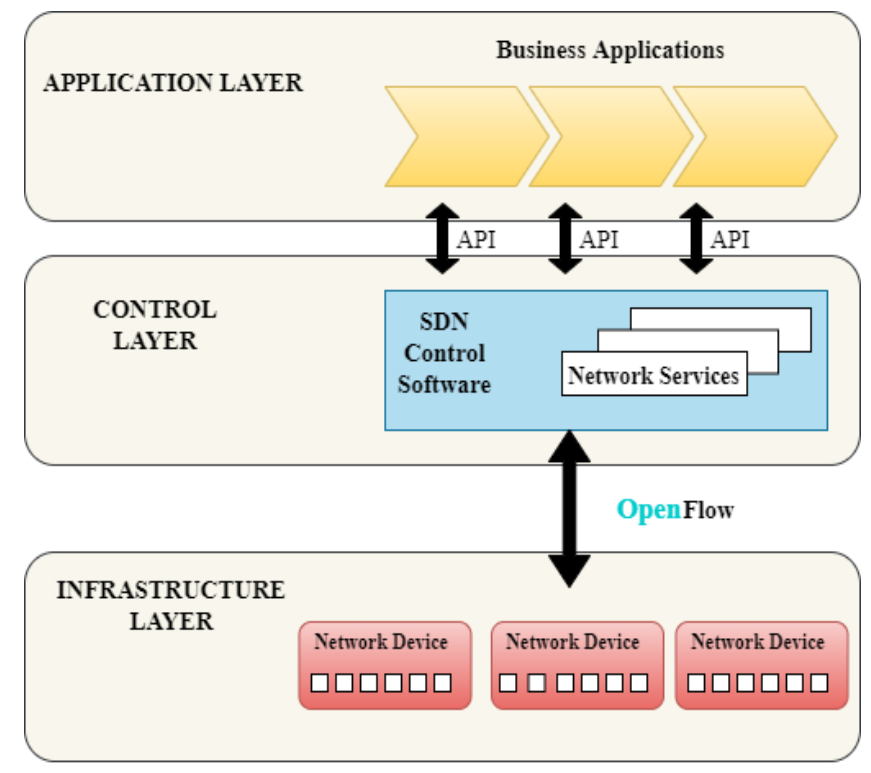

Figure 1. SDN architecture

\subsection{Routing protocol}

In this study, we compare the performance between interior routing protocols on SDN. We also tested exterior routing protocols with the same parameters to see the effects on the SDN. Interior routing protocols are OSPF and RIP and the exterior routing protocol is BGP. The selection of routing protocols is based on three types of algorithms, e.g. link state (for example OSPF), distance vectors (for example RIP), and path vectors (for example BGP). The BGP protocol delivers information packages between aoutonomous system (AS) [19]. AS is a set of routers that it is in the same administration with the same interior gateway protocol (IGP). The BGP is documented in RFC number 4271 [20]. OSPF is part of the IGP group that uses the Link-State protocol and the Djikstra algorithm which is more efficient than other IGP protocols [21]. In determining the best path, OSPF uses the smallest cost metric, namely the weighting of paths based on current conditions. The OSPF version 3 supports Ipv6 [22].

RIP is a distance-vector protocol that determining the best route by the number of hops. RIP has a simpler level of complexity, so that resource and memory consumption are also low. RIP is used on a small-scale network, with a few of hosts. RIPng (RIP next generation) is defined in RFC 2080, which is a RIPv2 development and IPv6 supporter [23].

\subsection{Quality of services}

In the process of analyzing SDN performance, several parameters will use in QoS measurements. Following is the Table 1, is the QoS value standard for multimedia services, there are voice call and video call on IP networks based on standard ITU-T G.1010.

Table 1. QoS value standard [24]

\begin{tabular}{cccc}
\hline \multicolumn{4}{c}{ Multimedia service parameter } \\
\hline Medium & Delay & Jitter & Packet loss \\
\hline Voice call & $<150 \mathrm{~ms}-<400 \mathrm{~ms}$ & $<1 \mathrm{~ms}$ & $<3 \%$ \\
Video call & $<150 \mathrm{~ms}-<400 \mathrm{~ms}$ & N/A & $<1 \%$ \\
\hline
\end{tabular}




\subsection{Mean opinion score}

Mean opinion score is a standard used to determine service quality based on user experience. The standard is divided into five levels called absolute category ranking (ACR) scale, which ranges from 1 to 5 [25]. The levels of the absolute category ranking are as in Figure 2. However, MOS measurements cannot be done in real-time. The objective measurement method proposed by Rec. ITU-T G.107 is called the E-model. It takes into account all interference factors that increase or decrease sound quality in one metric called the R-factor. R-factor is the measurement result of the E-model which is equivalent with MOS. The following formulas (1-5) are for obtaining MOS [26, 27].

$$
R=94.2-I_{d}-I_{e f}
$$

Where $I_{d}$ is a factor in the decrease in sound quality by a delay, $I_{e f}$ is a factor in quality loss due to compression and packet loss techniques.

$$
\begin{aligned}
& I_{d}=0.024 \times \mathrm{d}+0.11 \times(\mathrm{d}-177.3) \times \mathrm{H}(\mathrm{d}-177.3) \\
& I_{e f}=7+30 \times \ln (1+15 \mathrm{e})
\end{aligned}
$$

$\mathrm{R}$ is the value of delivery quality factor, $d$ is the delay value and $\mathrm{H}$ is the heavy side function and $e$ is a percentage of packet loss occurs.

$$
\mathrm{H}_{(\mathrm{x})}=\left\{\begin{array}{l}
0, \text { if } \mathrm{x}<0 \\
1, \text { if } \mathrm{x} \geq 0
\end{array}\right.
$$

Correlation between R-factor and ACR MOS, can be seen in (5) [28].

$$
\operatorname{MOS}=\left\{\begin{array}{c}
1, \text { if } R<0 \\
4.5, \text { if } R>100 \\
1+0.035 R+R(R-60)(100-R) 7 \times 10^{-6}, \text { if } 0<R<100
\end{array}\right.
$$

The $R$-factor refers to the MOS standard. The correlation among $R$-factor, ACR MOS and user satisfaction can be seen in Figure 2. Correlation is used to determine how good the quality of service produced is based on the level of user satisfaction.

$\begin{gathered}\text { Absolute Category Ranking } \\ \text { (ACR) MOS }\end{gathered}$
5. Excellent
4. Good
3. Fair
2. Poor
1. Bad

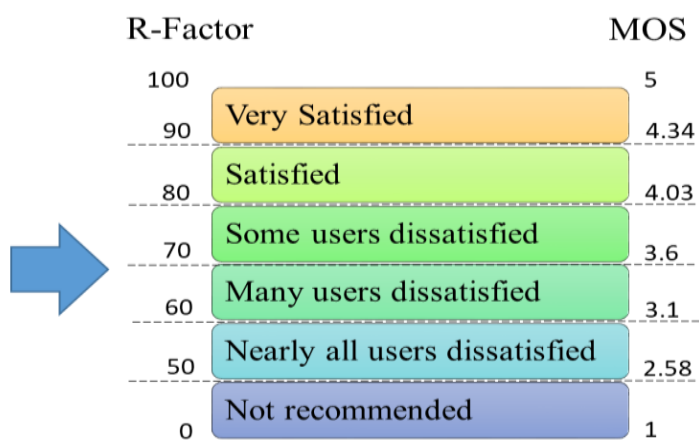

Figure 2. Correlation among $R$-factor, ACR MOS and user satisfaction

\section{SYSTEM MODEL}

\subsection{Network topology design}

The network topology design in this paper uses four computer, four switches and one conventional switch as a link as in Figure 3. The network topology consists of 4 hosts, 4 Open Vswitch (OVS) and 1 controller. Especially for the BGP protocol testing scenario, each PC is in a different AS. For software specifications (OS, simulator and Virtual Machine) and hardware (Computer user, Computer as controller, Switch) used can be seen in Table 2. The software design used RouteFlow which is installed on Ubuntu operating system that runs on a virtual machine (VM) using VMware. This RouteFlow has the capability of routing and switch control functions. 


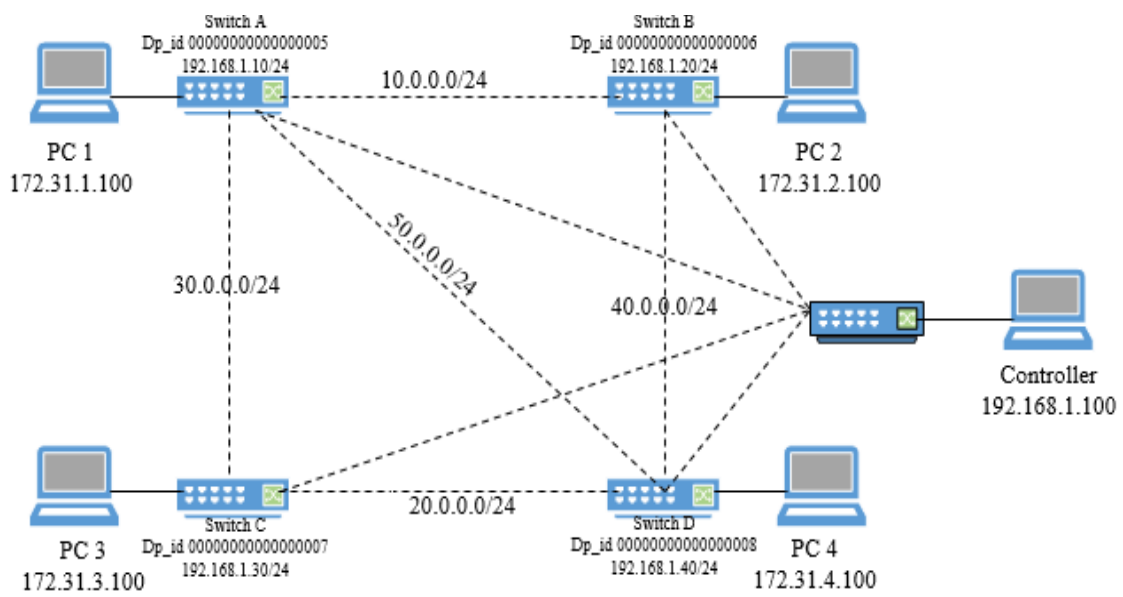

Figure 3. Network topology design

Table 2. Device specification

\begin{tabular}{ll}
\hline \multicolumn{1}{c}{ Component } & \multicolumn{1}{c}{ Specification } \\
\hline Computer as a controller & Intel(R) Core(TM) i3 CPU 400SU @ 1.7 GHz, RAM 6 GB, \\
Operating system (OS) & Ubuntu 12.04 LTS 64 bit (RouteFlow) \& Ubuntu 12.10 \\
& Quantal 64 bit (Mininet) \\
Forwarding plane & 5 Switch TP-Link WR1043ND v2, Frequency 2.4-2.4835Ghz \\
Virtual machine & 2 Vmware \\
Emulator & Mininet \\
Controller & POX controller on RouteFlow \\
Routing engine & Quagga \\
\hline
\end{tabular}

\subsection{Design of control system}

In the design of the control system the stage that carried out to design and make control SDN-based network. This RouteFlow installs on VM with Ubuntu operating system which has the ability to control routing functions. Process installation RouteFlow is to install POX controller, quagga, and supporting components of RouteFlow itself. The configuration stage on RouteFlow is in accordance with the network topology that has been designed before. There are also rfvm folders and files in the rftest folder that support RouteFlow environment. The following is an explanation for some of these files.

- rftest

rftest is a system configuration RouteFlow that integrates the components and files provided on the RouteFlow Environment that is used to run RouteFlow.

- rftest2config.csv

In the rftest2config.csv the implementation of configuration dp_id and dp_port on RouteFlow must be the same as the configurations datapath-id and Open vSwitch port on the OVS switch.

- daemons

The functions of the file daemons are to the enable routing protocol to be used. This research use routing protocols BGP, OSPF, and RIP, so it is activated bgpd, ospfd, and ripd.

\subsection{Design of forwarding}

Design of forwarding step is the steps taken to design and create a routing system of SDN-based network. The design was divided into two steps, there are installation OpenWrt and Open vSwitch and configuration port, the network topology using TP-Link WR1043ND wireless router. The device is installed with OpenWrt and Open vSwitch. OpenWrt used is OpenWrt version 15.05 Chaos Calmer which was built by compiling and implementing Open vSwitch version 2.3.90. Port Configuration on device forwarding is done by setting ports that is connected to controller, port configuration, and data plane port.

\subsection{Design of multimedia service}

In this stage is carried out to create a multimedia service used to connect between clients by installing Trixbox services on VM with the Linux operating system in Ubuntu version. As shown in Figure 4 We used IOS Trixbox version 2.8.0.4 and X-Lite version 5.5.0 is used for client communication. 


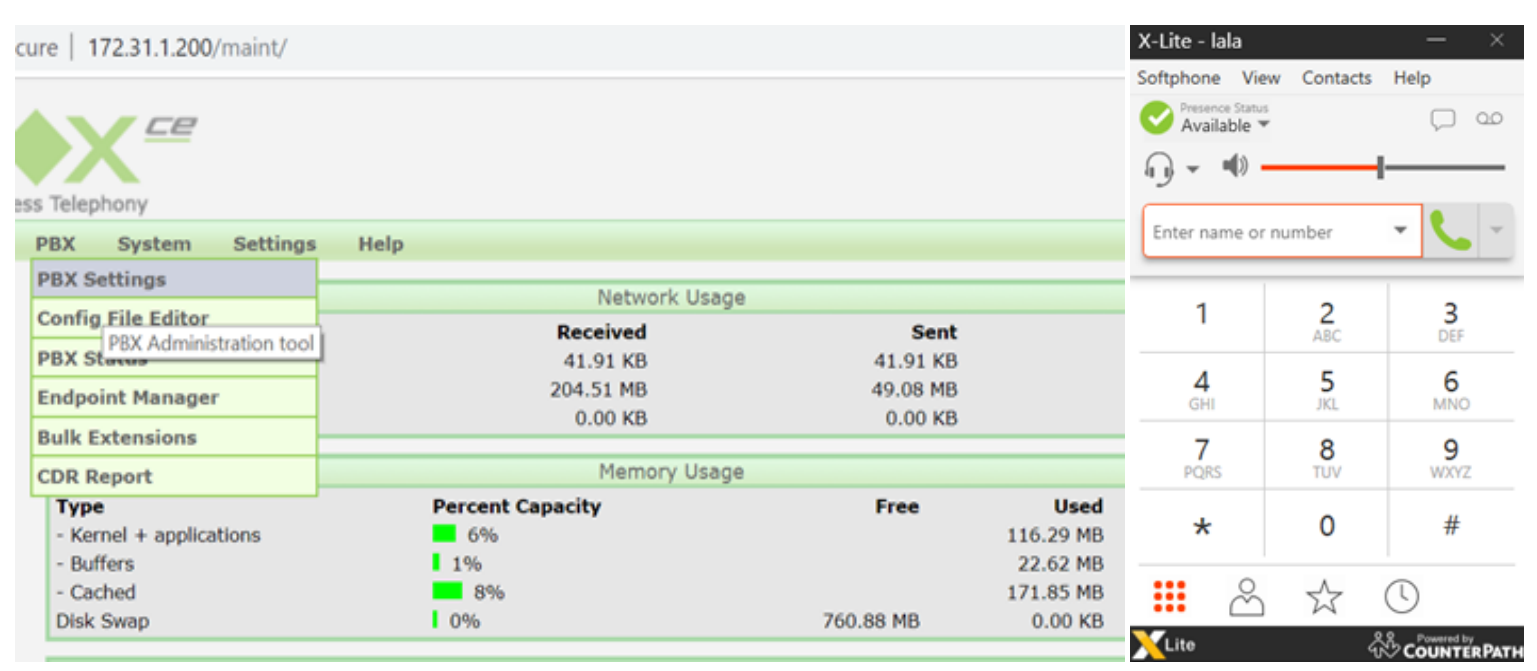

Figure 4. GUI of X-lite and Trixbox software

\section{RESULTS AND DISCUSSION}

\subsection{Performance measurement}

Performance measurement are implemented using several routing protocols such as BGP, OSPF, and RIP for multimedia services, there are voice call and video call using Wireshark. This test is carried out on PC 1 to PC 4 which is interconnected and can be assessed using several routing protocols with server-side (PC 1) on sender and client-side (PC 2) on the recipient. The measurements were carried out 10 times with each observation for 60 seconds. The parameters chosen are throughput, delay, jitter, convergence time and MOS.

\subsection{Comparison of $\mathrm{QoS}$ results}

The results QoS measurements on multimedia services were carried out, there are voice call and video call with background traffic in client side $10 \mathrm{Mbps}, 30 \mathrm{Mbps}, 50 \mathrm{Mbps}$, and $90 \mathrm{Mbps}$. The aims is to determine the server performance limits on traffic loads.

\subsubsection{Throughput}

Based on the test results, throughput carried out for 60 seconds, so the measurements results as in Figure 5, the difference in throughput value at each given traffic load. The average measurements obtained of voice call on routing BGP with background traffic $10 \mathrm{Mbps}$ is $59.8 \mathrm{Kbps}$, $30 \mathrm{Mbps}$ is $55.9 \mathrm{Kbps}$, $50 \mathrm{Mbps}$ is $42.3 \mathrm{Kbps}$, and $90 \mathrm{Mbps}$ is $6.7 \mathrm{Kbps}$, routing OSPF with background traffic $10 \mathrm{Mbps}$ is $60.7 \mathrm{Kbps}, 30 \mathrm{Mbps}$ is $57.7 \mathrm{Kbps}$, $50 \mathrm{Mbps}$ is $43.8 \mathrm{Kbps}$, and $90 \mathrm{Mbps}$ is $8.7 \mathrm{Kbps}$, and routing RIP with background traffic $10 \mathrm{Mbps}$ is $58.8 \mathrm{Kbps}, 30 \mathrm{Mbps}$ is $54.6 \mathrm{Kbps}, 50 \mathrm{Mbps}$ is $41.8 \mathrm{Kbps}$, and $90 \mathrm{Mbps}$ is $5.2 \mathrm{Kbps}$.

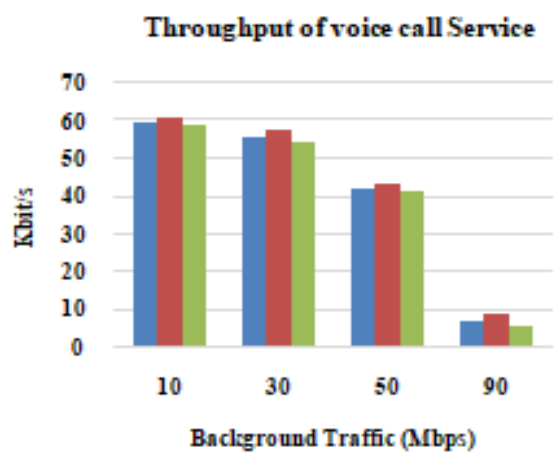

(a)

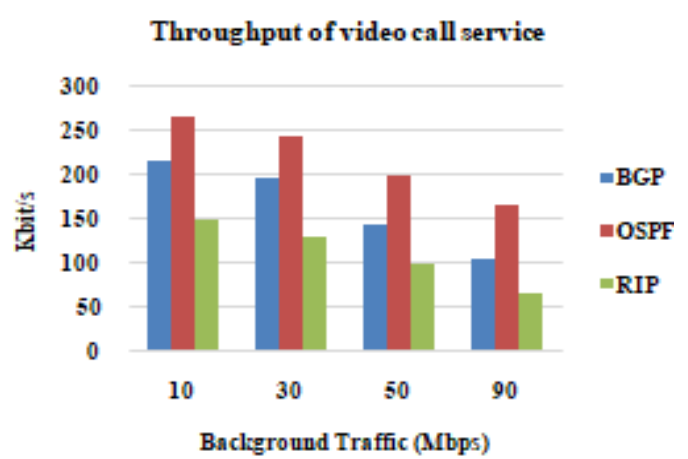

(b)

Figure 5. Throughput with background traffic of (a) voice call, (b) video call

In Figure 5, the average measurement throughput of video call on routing BGP with background traffic $10 \mathrm{Mbps}$ is $214.7 \mathrm{Kbps}, 30 \mathrm{Mbps}$ is $196.4 \mathrm{Kbps}, 50 \mathrm{Mbps}$ is $143.9 \mathrm{Kbps}$, and $90 \mathrm{Mbps}$ is $102.7 \mathrm{Kbps}$, routing OSPF with background traffic $10 \mathrm{Mbps}$ is $265.5 \mathrm{Kbps}, 30 \mathrm{Mbps}$ is $243.5 \mathrm{Kbps}, 50 \mathrm{Mbps}$ is 198.9 Kbps, and $90 \mathrm{Mbps}$ is $165.8 \mathrm{Kbps}$, and routing RIP with background traffic $10 \mathrm{Mbps}$ is $147.7 \mathrm{Kbps}$, 
$30 \mathrm{Mbps}$ is $128.8 \mathrm{Kbps}, 50 \mathrm{Mbps}$ is $97.6 \mathrm{Kbps}$, and $90 \mathrm{Mbps}$ is $64.9 \mathrm{Kbps}$. In Figure 5, if traffic load is given large, value throughput will be smaller. This happen because heavy traffic so the available bandwidth is also getting denser and the number of bits sent every second decreases.

\subsubsection{Delay}

Based on testing that has been done for 60 seconds, the results average delay of voice call can be seen as in Figure 5 on routing BGP with background traffic $10 \mathrm{Mbps}$ is $19.565 \mathrm{~ms}, 30 \mathrm{Mbps}$ is $20.060 \mathrm{~ms}$, $50 \mathrm{Mbps}$ is $21.065 \mathrm{~ms}$, and $90 \mathrm{Mbps}$ is $21.474 \mathrm{~ms}$, routing OSPF with background traffic $10 \mathrm{Mbps}$ is 19.438 $\mathrm{ms}$, $30 \mathrm{Mbps}$ is $19.754 \mathrm{~ms}$, $50 \mathrm{Mbps}$ is $20.389 \mathrm{~ms}$, and $90 \mathrm{Mbps}$ is $21.094 \mathrm{~ms}$, and routing RIP with background traffic $10 \mathrm{Mbps}$ is $19.780 \mathrm{~ms}, 30 \mathrm{Mbps}$ is $20.438 \mathrm{~ms}, 50 \mathrm{Mbps}$ is $21.205 \mathrm{~ms}$, and $90 \mathrm{Mbps}$ is $22.037 \mathrm{~ms}$. In Figure 6, the average measurements delay of video call on routing BGP with background traffic $10 \mathrm{Mbps}$ is $19.9981 \mathrm{~ms}, 30 \mathrm{Mbps}$ is $20.230 \mathrm{~ms}, 50 \mathrm{Mbps}$ is $21.247 \mathrm{~ms}$, and $90 \mathrm{Mbps}$ is $22.342 \mathrm{~ms}$, routing OSPF with background traffic $10 \mathrm{Mbps}$ is $19.565 \mathrm{~ms}, 30 \mathrm{Mbps}$ is $19.874 \mathrm{~ms}, 50 \mathrm{Mbps}$ is $21.237 \mathrm{~ms}$, and $90 \mathrm{Mbps}$ is $22.858 \mathrm{~ms}$, and routing RIP with background traffic $10 \mathrm{Mbps}$ is $20.081 \mathrm{~ms}, 30 \mathrm{Mbps}$ is $22.497 \mathrm{~ms}$, and $50 \mathrm{Mbps}$ is $24.154 \mathrm{~ms}$, and $90 \mathrm{Mbps}$ is $25.172 \mathrm{~ms}$.

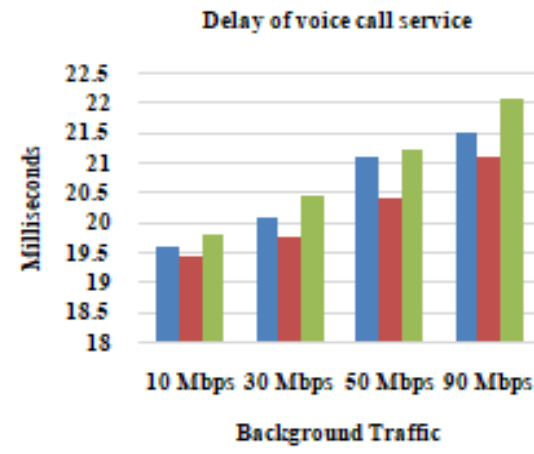

(a)

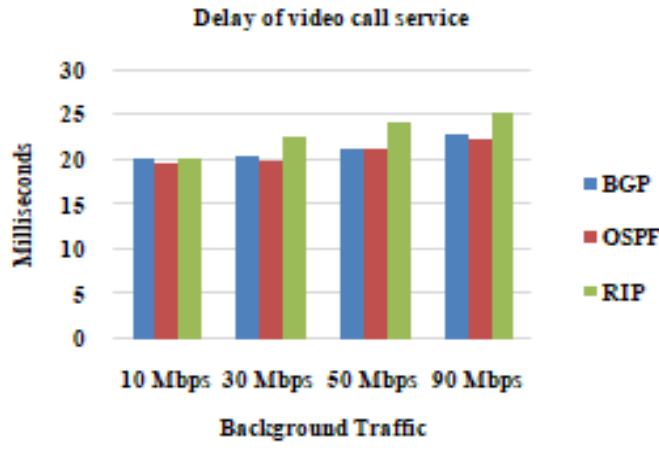

(b)

Figure 6. The delay with background traffic of (a) Voice call, (b) Video call

\subsubsection{Jitter}

Based on testing carried out for 60 seconds, the average measurements of voice call can be seen in Figure 7, on routing BGP with background traffic is $0.254 \mathrm{~ms}, 30 \mathrm{Mbps}$ is $0.340 \mathrm{~ms}, 50 \mathrm{Mbps}$ is $0.382 \mathrm{~ms}$, and $90 \mathrm{Mbps}$ is $0.437 \mathrm{~ms}$, routing OSPF with background traffic $10 \mathrm{Mbps}$ is $0.224 \mathrm{~ms}, 30 \mathrm{Mbps}$ is $0.254 \mathrm{~ms}$, $50 \mathrm{Mbps}$ is $0.315 \mathrm{~ms}$, and $90 \mathrm{Mbps}$ is $0.431 \mathrm{~ms}$, and routing RIP with background traffic $10 \mathrm{Mbps}$ is 0.437 $\mathrm{ms}, 30 \mathrm{Mbps}$ is $0.689 \mathrm{~ms}$, and $50 \mathrm{Mbps}$ is $0.744 \mathrm{~ms}$, and $90 \mathrm{Mbps}$ is $0.753 \mathrm{~ms}$. In Figure 7, the average measurement jitter of video call on routing BGP with background traffic $10 \mathrm{Mbps}$ is $0.529 \mathrm{~ms}, 30 \mathrm{Mbps}$ is $0.603 \mathrm{~ms}, 50 \mathrm{Mbps}$ is $0.382 \mathrm{~ms}$, and $90 \mathrm{Mbps}$ is $0.437 \mathrm{~ms}$, routing OSPF with background traffic $10 \mathrm{Mbps}$ is $0.312 \mathrm{~ms}, 30 \mathrm{Mbps}$ is $0.365 \mathrm{~ms}, 50 \mathrm{Mbps}$ is $0.545 \mathrm{~ms}$, and $90 \mathrm{Mbps}$ is $0.786 \mathrm{~ms}$, and routing BGP $10 \mathrm{Mbps}$ is $0.884 \mathrm{~ms}, 30 \mathrm{Mbps}$ is $0.955 \mathrm{~ms}, 50 \mathrm{Mbps}$ is $1.062 \mathrm{~ms}$, and $90 \mathrm{Mbps}$ is $1.254 \mathrm{~ms}$. In Figure 7 can be seen increasing the traffic background, then the variation on delay (jitter) is also getting bigger. The higher the background traffic, the greater the utility of the link which causes the jitter value to vary and be unstable.

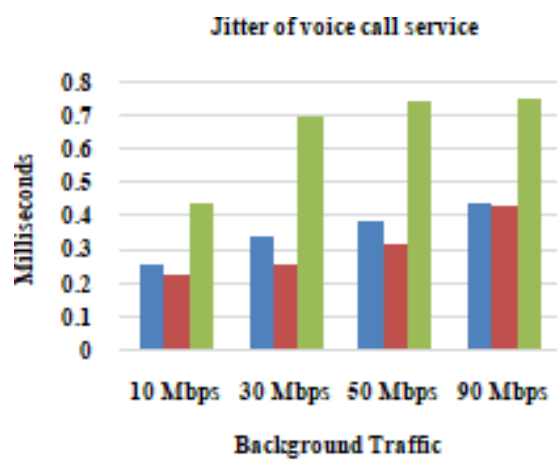

(a)

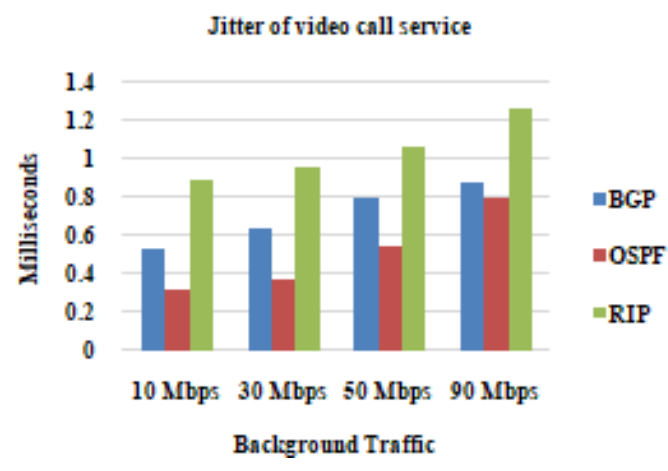

(b)

Figure 7. The jitter with background traffic of (a) voice call, (b) video call 


\subsubsection{Convergence time}

The convergence time measurement aims, to determine time needed by the router to reach the state of convergence. The following graph convergence time on SDN-based computer networks of simulation and implementation methods by performing 10 times the experiment. In Figure 8, it can be concluded that convergence time of simulation and implementation is not much different. The convergence time results of simulation with routing BGP is $11.3 \mathrm{~s}$, routing OSPF is $4.1 \mathrm{~s}$, and routing RIP is $14.9 \mathrm{~s}$. The implementation with routing BGP is $13.1 \mathrm{~s}$, routing OSPF is $4.3 \mathrm{~s}$, and routing RIP is $15.1 \mathrm{~s}$. The less convergence time, the better the network.

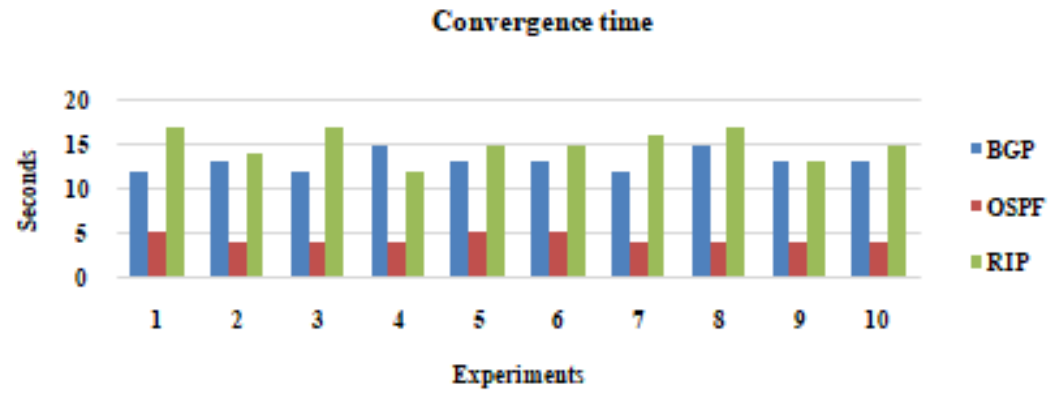

Figure 8 . The time convergence time

\subsubsection{MOS}

MOS measurements are carried out within 60 seconds. MOS values are determined based on (1)-(5). The average MOS on voice call with routing BGP is 4.08, OSPF is 4.13, and RIP is 4.01. And the average MOS on video call with routing BGP is 4.05 , OSPF, 4.11, and RIP is 4.02 . At Figure 9 can be concluded the average value MOS on video call and voice call are called Good quality.

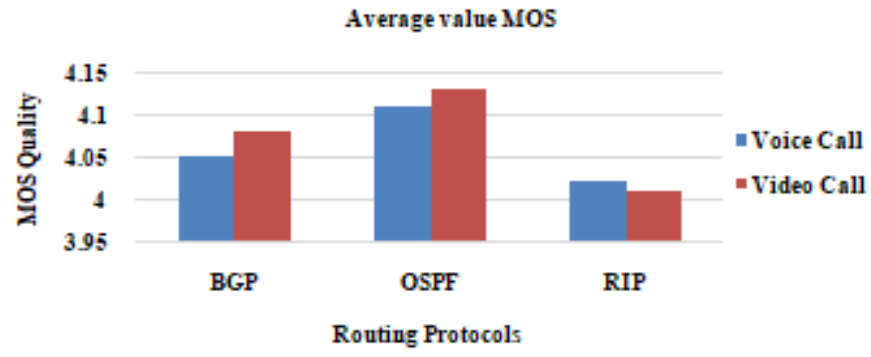

Figure 9. MOS average value of voice call and video call

\section{CONCLUSION}

In this study, we successfully applied the SDN concept to separate the control plane and the data plane on conventional network devices. We implement the control plane and data plane on the switch using open Vswitch and route flow. SDN networks that are built can serve multimedia applications well. Test results between the OSPF and RIP protocols for voice and video call services based on parameters throughput, delay, jitter, convergence time and MOS show that OSPF produces the best performance. Testing on the BGP protocol with its exterior characteristics has the same impact as other protocols, the greater the background traffic, the lower the output. The effect of adding background traffic from $10 \mathrm{Mbps}$ to $90 \mathrm{Mbps}$ reduces the QoS value, although it is still in a good category based on Rec. ITU-T G.1010, G.107, and P.910.

\section{REFERENCES}

[1] R. Tulloh, H. Tussyadiah, R. W. Hutabri and M. Negara, "Load distribution analysis on bipartite," Journal of Theoretical and Applied Information Technology, vol. 96, no. 5, pp. 1238-1252, 2018.

[2] P. E. Numan et al., "On the latency and jitter evaluation of software defined networks," Bulletin of Electrical Engineering and Informatics, vol. 8, no. 4, pp. 1507-1516, 2019. 
[3] S. Jain et al., "B4: Experience with a globally-deployed software defined WAN," ACM SIGCOMM Computer Communication Review, vol. 43, no. 4, pp. 3-14, 2013.

[4] S. Singh and R. K. Jha, "A survey on software defined networking: Architecture for next generation network," Journal of Network and Systems Management, vol. 25, no. 2, pp. 321-374, 2017.

[5] M. Karakus and A. Durresi, "Quality of service (QoS) in software defined networking (SDN): A survey," Journal of Network and Computer Applications, vol. 80, pp. 20-218, 2017.

[6] N. McKeown et al., "OpenFlow: Enabling innovation in campus networks," ACM SIGCOMM Computer Communication Review, vol. 38, no. 2, pp. 69-74, 2008.

[7] M. Alsaeedi, M. M. Mohamad, and A. A. Al-Roubaiey, "Toward adaptive and scalable OpenFlow-SDN flow control: A survey," IEEE Access, vol. 7, pp. 107346-107379, 2019.

[8] R. M. Negara and R. Tulloh, "Simulation analysis of OSPF algorithm application using routeflow on software defined network (SDN)," in Bahasa: Analisis simulasi penerapan algoritma ospf menggunakan routeflow pada jaringan software defined network (SDN)," Journal of Informatics, Telecommunication and Electronics (INFOTEL), vol. 9, no. 1, pp. 75-83, 2017.

[9] A. A. Khan, M. Zafrullah, M. Hussain, and A. Ahmad, "Performance analysis of OSPF and hybrid networks," International Symposium on Wireless Systems and Networks (ISWSN), vol. 2018, pp. 1-4, 2018.

[10] R. Adrian, A. Dahlan, and K. Anam, "OSPF cost impact analysis on SDN network," 2nd Int. Conf. on Information Technology, Information Systems and Electrical Engineering (ICITISEE), vol. 2018, pp. 198-201, 2018.

[11] S. Abdallah, A. Kayssi, I. H. Elhajj, and A. Chehab, "Network convergence in SDN versus OSPF networks," 5th International Conference on Software Defined Systems, pp. 130-137, 2018.

[12] L. M. Elguea and F. Martinez-Rios, "A new method to optimize BGP routes using SDN and reducing latency," Procedia Computer Science, vol. 135, pp. 163-169, 2018.

[13] P. Sermpezis and X. Dimitropoulos, "Can SDN accelerate BGP convergence? - A performance analysis of interdomain routing centralization," IFIP Net. Conference (IFIP Networking) and Workshops, vol. 2018, pp. 1-9, 2017.

[14] V. Baggan and S. N. Panda, "Enhancing border gateway routing protocol with software defined networking," International Journal of Innovative Technology and Exploring Engineering, vol. 8, no. 8, pp. 976-984, 2019.

[15] L. Han, S. Sun, B. Joo, X. Jin, and S. Han, "QoS-aware routing mechanism in OpenFlow-enabled wireless multimedia sensor networks," International Journal of Distributed Sensor Networks, vol. 12, no. 7, pp. 1-11, 2016.

[16] D. Gopi, S. Cheng, and R. Huck, "Comparative analysis of SDN and conventional networks using routing protocols," International Conference on Computer, Information and Telecommunication Systems (CITS), pp. 108-112, 2017.

[17] E. Amiri, M. R. Hashemi, and K. R. Lejjy, "Policy-based routing in RIP-hybrid network with SDN controller," 4th National Conference on Applied Research Electrical Mechanical Computer and IT Engineering, pp. 1-8, 2018.

[18] F. Hu, Q. Hao, and K. Bao, "A survey on software-defined network and OpenFlow: From concept to implementation," IEEE Communications Surveys \& Tutorials, vol. 16, no. 4, pp. 2181-2206, 2014

[19] B. Al-Musawi, P. Branch, and G. Armitage, "BGP anomaly detection techniques: A survey," IEEE Comm. Surveys \& Tutorials, vol. 19, no. 1, pp. 377-396, 2017.

[20] Y. Rekhter, T. Li, and S. Hares, "RFC 4271, a border gateway protocol 4 (BGP-4)," IETF Datatracker, 2006.

[21] M. Jayakumar, N. R. S. Rekha, and B. Bharathi, "A comparative study on RIP and OSPF protocols," International Conference on Innovations in Information, Embedded and Communication Systems, pp. 1-5, 2015.

[22] R. Coltun, D. Ferguson, and J. Moy, "RFC5340: OSPF for IPv6," Network Working Group, IETF, pp. 1-94, 2008.

[23] G. Malkin and R. Minnear, "RFC 2080: RIPng for IPv6," Network Working Group, IETF, pp. 1-19, 1997.

[24] ITU-T Recommendation G.1010, "Series G: Transmission systems and media, digital systems and networs: Quality of service and performance: End-user multimedia QoS categories," International Telecommunication Union, pp. 1-18, 2001.

[25] Recommendation ITU-T P.910, "Series P: Telephone Transmission Quality, Telephone Instalations, local Line Networks: Audiovisual quality in multimedia services: Subjective video quality assessment methods for multimedia applications," International Telecommunication Union, pp. 1-42, 2008.

[26] D-H. Nguyen, H. Nguyen, and E. Renault, "A new non-intrusive assessment method for VoLTE quality based on extended e-model," International Conference on Mobile, Secure, and Programmable Networking. Springer, Cham, pp. 122-136, 2016.

[27] C. Olariu, M. Foghlu, P. Perry, and L. Murphy, "VoIP quality monitoring in LTE femtocells," IFIP/IEEE International Symposium on Integrated Network Management, pp. 501-508, 2011.

[28] Recommendation ITU-T G.107, "Series G: Transmission systems and media, digital systems and networks: International telephone connections and circuits-transmission planning and the E-model: The E-model: A computational model for use in transmission planning," International Telecommunication Union, pp. 1-30, 2015. 